\title{
AN OBJECT-ORIENTED RANDOM-NUMBER PACKAGE WITH MANY LONG STREAMS AND SUBSTREAMS
}

\author{
PIERRE L'ECUYER and RICHARD SIMARD \\ Département d'informatique et de recherche opérationnelle, Université de Montréal, C.P. 6128 \\ succ. Centre-Ville, Montréal, Québec H3C 3J\%, Canada \\ lecuyer@iro.umontreal.ca•simardr@iro.umontreal.ca \\ E. JACK CHEN \\ BASF Corporation, 3000 Continental Drive-North, Mount Olive, New Jersey 07828-1234, USA \\ chenej@basf.com

\section{W. DAVID KELTON} \\ Department of Management Science and Information Systems, The Smeal College of Business \\ Administration, 303 Beam, The Pennsylvania State University, University Park, PA 16802-1913, \\ USA \\ dkelton@psu.edu
}

(Received December 2000)

Multiple independent streams of random numbers are often required in simulation studies, for instance, to facilitate synchronization for variance-reduction purposes, and for making independent replications. A portable set of software utilities is described for uniform randomnumber generation. It provides for multiple generators (streams) running simultaneously, and each generator (stream) has its sequence of numbers partitioned into many long disjoint contiguous substreams. The basic underlying generator for this implementation is a combined multiple recursive generator with period length of approximately $2^{191}$, proposed in a previous paper. A $\mathrm{C}++$ interface is described here. Portable implementations are available in $\mathrm{C}$, $\mathrm{C}++$, and Java via the On-line Companion to this paper on the Operations Research website.

This report is an expanded version of the article by L'Ecuyer et al. (2001).

Subject classifications: Simulation: Random number generation, Simulation: Random variable generation, Simulation: Statistical analysis, Computers/computer science: Software

Experts now recognize that small linear congruential generators (LCGs) with moduli around $2^{31}$ or so should no longer be used as general-purpose random-number generators (RNGs). Not only can one exhaust the period in a few minutes on a PC, but more importantly the poor structure of the points can dramatically bias simulation results for sample sizes much smaller than the period length.

As an illustration, we consider a variant of the birthday problem, defined as follows. Partition the unit square $[0,1)^{2}$ into $k$ cells (square boxes) of equal sizes. Identify these cells as 0 through $k-1$. If we generate $2 n$ random numbers $U_{0}, \ldots, U_{2 n-1}$, then each non-overlapping 2-tuple $V_{i}=\left(U_{2 i}, U_{2 i+1}\right), i=0, \ldots, n-1$, will fall into some cell $I_{i} \in\{0, \ldots, k-1\}$. Let 


\section{Table I}

\begin{tabular}{ccc}
$p$-values for the birthday proble \\
\hline$n$ & $y$ & $p^{+}(y)$ \\
\hline $2^{12}$ & 2 & 0.26 \\
$2^{13}$ & 16 & $1.9 \times 10^{-14}$ \\
$2^{14}$ & 170 & $<10^{-15}$ \\
$2^{15}$ & 1310 & $<10^{-15}$ \\
$2^{16}$ & 10060 & $<10^{-15}$ \\
$2^{17}$ & 53907 & $<10^{-15}$ \\
\hline
\end{tabular}

$I_{(1)} \leq I_{(2)} \leq \cdots \leq I_{(n)}$ be the identification of the cells sorted by increasing order. Compute the spacings $S_{j}=I_{(j+1)}-I_{(j)}$, for $j=1, \ldots, n-1$, and let $Y$ be the number of values of $j \in\{1, \ldots, n-2\}$ such that $S_{(j+1)}=S_{(j)}$, where $S_{(1)}, \ldots, S_{(n-1)}$ are the spacings sorted by increasing order. These $n$ cells can be viewed as the birthdays (between day 0 and day $k-1$ ) of $n$ people in a world where years have $k$ days, whence the name birthday spacings (Marsaglia 1985, L'Ecuyer and Simard 2001). Suppose we want to estimate $E[Y]$ by simulation, using the LCG:

$$
x_{i}=a x_{i-1} \bmod m, \quad u_{i}=x_{i} / m, \quad x_{0} \in\{1, \ldots, m-1\},
$$

with $a=16807$ and $m=2^{31}-1$. This LCG is widely used. We made ten replications of the simulation, with $n=2^{15}=32768$ and $k \approx 2^{43}$, and obtained ten values of $Y$ ranging from 1315 to 1404 . (Each replication takes $<0.02 \mathrm{sec}$ on a PC.) One may then conclude that $E[Y]$ is somewhere between 1300 and 1400. In fact, it can be shown (theoretically) that $Y$ has approximately the Poisson distribution with mean $\lambda=n^{3} /(4 k)$ when $n$ is large and $\lambda$ is small (Marsaglia 1985). Here, we took $k \approx n^{3} / 4$, so $E[Y]=\lambda \approx 1$. The above estimate is thus very far from the truth! If $Y$ takes the value $y$, the (right) $p$-value is $p^{+}(y)=P\left[Y \geq y \mid H_{0}\right]$, where $H_{0}$ is the null hypothesis that $E(Y)=y$. Table I gives the estimated value $y$ of $E(Y)$, and the corresponding $p$-value, with different values of $n$ and with $k \approx n^{3} / 4$. In all cases, the true distribution of $Y$ is approximately Poisson with mean 1 . For $n \geq 2^{13}$, the $p$-values are virtually zero, i.e., the estimates are highly inaccurate. Changing the multiplier $a$ brings no significant improvement: All LCGs start to fail at $n \approx 8 m^{1 / 3}$. The problem is that the structure of the points is much too regular. We clearly need better RNGs!

Much better RNGs have already been proposed to replace older unsafe LCGs. We mention, for instance, the Mersenne twister of Matsumoto and Nishimura (1998), the combined 
MRGs of L'Ecuyer (1999a), the combined LCGs of L'Ecuyer and Andres (1997), and the combined Tausworthe generators of L'Ecuyer (1999b). All of these have fairly solid theoretical support, have been extensively tested, and are easy to use.

However, a single RNG does not always suffice. Many disjoint random-number subsequences, each having long period and good statistical properties, are often required in simulation studies, for instance, to make independent replications or to associate distinct "streams" of random numbers with different sources of randomness in the system to facilitate synchronization for variance reduction (Law and Kelton 2000). Suppose, for instance, that one wants to perform independent pairs of replications with common random numbers across the configurations (i.e., between any two replications of the same pair) in order to compare two different competing configurations of a system. To ensure proper synchronization, we want every generator to start from the same seed in both runs of the same pair. However, in general, these two runs will make a different number of calls to a generator, and programming "tricks" have to be used to skip a proper amount of random numbers to resynchronize the generators for the next pair without overlap in the random-number streams. This requires extra programming effort and is error-prone. Good software tools should ease the programmer's task in that respect (L'Ecuyer and Côté 1991).

In this paper, we propose a package for uniform random-number generation with multiple streams of (pseudo)random numbers and convenient tools to move around within and across these streams. The structure and the tools offered are similar to those in the package proposed by L'Ecuyer and Côté (1991) and L'Ecuyer and Andres (1997). The main differences are:

- The underlying "backbone" generator is more robust and has longer period than those used by these authors. We use the combined multiple recursive generator (CMRG) MRG32k3a proposed by L'Ecuyer (1999a).

- The package proposed here has an object-oriented design. The streams, which can be seen as virtual RNGs, are declared at will, as instances of a class, instead of being numbered from 0 to $N$ where $N$ is fixed.

- The generator is implemented in floating-point arithmetic instead of integer arithmetic. This is faster on most current 32-bit computers, for which 64-bit floating-point operations are implemented in hardware on the CPU. 
Other random number packages with multiple streams have been proposed in recent years; see for example Mascagni and Srinivasan (2000). These packages do not offer the same tools for streams and substreams as ours, and are not supported by the same theoretical analysis for the quality and independence of the different streams.

The rest of this paper is organized as follows. Section 1 provides some background on the backbone CMRG and explains the idea of multiple streams and substreams. In Section 2, we describe support utilities. We give a quick and easy way to compute $a \times s$ mod $m$ for any positive integer values of $a<m, s<m$, and $m<2^{32}$, for computers that support the IEEE754 standard (which ensures that floating-point numbers have at least 53 bits of precision for the mantissa). We also describe a code to compute $A^{v} \bmod m$, where $A$ is a square matrix of integers. These kinds of computations are required for jumping by several steps at a time in the generator's sequence (e.g., for jumping to distant streams or substreams). Section 3 describes a package with multiple streams and substreams, and gives the interface of its $\mathrm{C}++$ implementation. Section 4 provides some examples of using this RNG package. Section 5 summarizes. The appendix gives the complete $\mathrm{C}++$ implementation. Implementations in $\mathrm{C}$ and Java are available at http://www.iro.umontreal.ca/ lecuyer.

\section{DESCRIPTION AND IMPLEMENTATION OF THE SOFTWARE}

\subsection{The underlying backbone generator}

L'Ecuyer (1999a) gave several good parameter sets for CMRGs of different sizes. We have selected one of them, called MRG32k3a, as our backbone generator. It has 2 components

each of order 3. At step $n$, its state is the pair of vectors $s_{1, n}=\left(x_{1, n}, x_{1, n+1}, x_{1, n+2}\right)$ and $s_{2, n}=\left(x_{2, n}, x_{2, n+1}, x_{2, n+2}\right)$, which evolve according to the linear recurrences

$$
\begin{aligned}
& x_{1, n}=\left(1403580 \times x_{1, n-2}-810728 \times x_{1, n-3}\right) \bmod m_{1} \\
& x_{2, n}=\left(527612 \times x_{2, n-1}-1370589 \times x_{2, n-3}\right) \bmod m_{2},
\end{aligned}
$$

where $m_{1}=2^{32}-209=4294967087$ and $m_{2}=2^{32}-22853=4294944443$, and its output $u_{n}$ is defined by

$$
\begin{aligned}
& z_{n}=\left(x_{1, n}-x_{2, n}\right) \bmod 4294967087 \\
& u_{n}= \begin{cases}z_{n} / 4294967088 & \text { if } z_{n}>0 \\
4294967087 / 4294967088 & \text { if } z_{n}=0\end{cases}
\end{aligned}
$$


Its period length is $\rho=\left(m_{1}^{3}-1\right)\left(m_{2}^{3}-1\right) / 2 \approx 2^{191} \approx 3.1 \times 10^{57}$. RNGs with much longer periods are also available, but their states must contain more bits and are therefore more expensive to manipulate. We think that our choice is a reasonable compromise. The parameters have been chosen so that the period is long, a fast implementation is available (in floating point arithmetic), and the generator performs well with respect to the spectral test in up to (at least) 45 dimensions. The spectral test in $t$ dimensions measures the uniformity of the point set

$$
T_{t}=\left\{\left(u_{0}, \ldots, u_{t-1}\right) \mid\left(x_{1,0}, x_{1,1}, x_{1,2}\right) \in \mathbf{Z}_{m_{1}}^{3},\left(x_{2,0}, x_{2,1}, x_{2,2}\right) \in \mathbf{Z}_{m_{2}}^{3}\right\}
$$

where $\mathbf{Z}_{m}=\{0, \ldots, m-1\}$, and makes sure that this set covers the $t$-dimensional unit hypercube very uniformly. This $T_{t}$ is the set of all overlapping $t$-tuples of successive values produced by the generator, from all possible initial states. It turns out to be the intersection of a lattice with the $t$-dimensional unit hypercube. This means that all the points of $T_{t}$ lie in a limited number of equidistant parallel hyperplanes. The parameters of the generator were chosen so that the distance between the successive hyperplanes is close to the minimal achievable distance given the total number of points $m_{1} m_{2}$, for all $t$ up to 45 .

\subsection{Multiple streams and substreams}

Let $\rho$ be the period length of the RNG and $T$ its transition function; that is, $T\left(s_{n}\right)=s_{n+1}$ where $s_{n}$ is the generator's state at step $n$, and $T^{\rho}(s)=s$. To partition the generator's sequence into disjoint streams and substreams, we choose two positive integers $v$ and $w$, and let $z=v+w$. We first cut the long cycle into adjacent streams of length $Z=2^{z}$ and then partition each of these streams into $V=2^{v}$ blocks (or substreams) of length $W=2^{w}$.

If $s_{0}$ is the initial seed of the generator and $I_{g}$ denotes the initial state of stream $g$ for $g \geq 1$, then we have $I_{1}=s_{0}, I_{2}=T^{Z}\left(s_{0}\right), \ldots, I_{g}=T^{Z}\left(I_{g-1}\right)=T^{(g-1) Z}\left(s_{0}\right), \ldots$ The first substream of stream $g$ starts in state $I_{g}$, the second one in state $T^{W}\left(I_{g}\right)$, the third one in state $T^{2 W}\left(I_{g}\right)$, and so on. At any moment during program execution, stream $g$ is in some state, say $C_{g}$. We denote by $B_{g}$ the starting state of the substream that contains the current state, i.e., of the current substream, and $N_{g}=T^{W}\left(B_{g}\right)$ the starting state of the next substream. In the following illustration, for example, the state of stream $g$ is at the $6^{\text {th }}$ value of the third substream, i.e., $2 W+5$ steps ahead of its initial state $I_{g}$ and 5 steps ahead of $B_{g}$. Figure 1 depicts the overall arrangement of the streams and substreams, and indicates the spacings and number of each. 


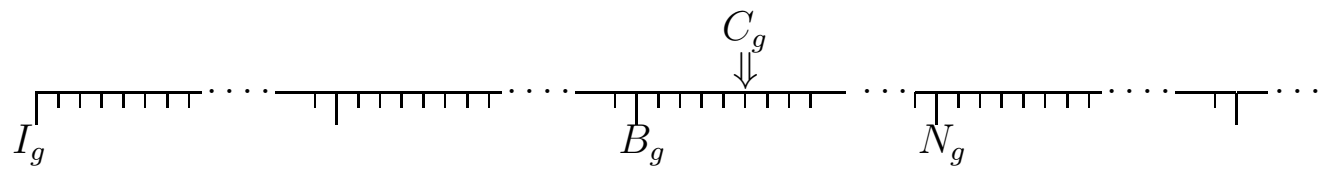

Whenever a new stream is created (instantiated), say the $g^{\text {th }}$ stream, the software automatically computes $I_{g}=T^{Z}\left(I_{g-1}\right)$ and puts $C_{g}=B_{g}=I_{g}$. When going from a substream to the next one, the software must compute $N_{g}=T^{W}\left(B_{g}\right)$. Of course, $W$ and $Z$ must be huge numbers, so a quick way to compute $s_{n+\nu}$ from $s_{n}$ for large integers $\nu$, without generating the intermediate values, must be available. For a combined MRG, we can do this for each of its components separately, as explained in L'Ecuyer (1990): one can write $s_{j, n+1}=A_{j} s_{j, n} \bmod m_{j}$ for some $3 \times 3$ matrix $A_{j}$, and then $s_{j, n+\nu}=\left(A_{j}^{\nu} \bmod m_{j}\right) s_{j, n} \bmod m_{j}$, see Section 2.2. The matrix $A_{j}^{\nu} \bmod m_{j}$ is computed via a standard divide-and-conquer algorithm (Knuth 1998), and can be precomputed once for $\nu=W$ and $\nu=Z$. Most other random-number packages offer no facility for jumping ahead directly from $s_{n}$ to $s_{n+\nu}$ or to compute distant seeds efficiently.

\subsection{Choice of $v$ and $w$}

We have selected $v=51$ and $w=76$, so $W=2^{76}$ and $Z=2^{127}$. To select $v$ and $w$, a spectral test for the vectors of non-successive output values spaced $h=2^{l}$ steps apart was performed for different integer values of $l$, and we chose $v$ and $w$ so that the behavior was good for $l=v, l=w$, and $l=v+w$. More specifically, let $T_{t}(s, h)$ be the point set obtained if we replace $\left(u_{n}, \ldots, u_{n+t-1}\right)$ by the first $t$ components of the sequence $\left(u_{n}, \ldots, u_{n+s-1}, u_{h}, \ldots, u_{n+h+s-1}, u_{n+2 h}, \ldots, u_{n+2 h+s-1}, \ldots\right)$ in the definition of $T_{t}$. If the streams are started $h$ apart, the points of $T_{t}(s, h)$ are those obtained by taking $s$ successive values from the first stream, $s$ successive values from the second stream, and so on until $t$ values have been taken. These points have a lattice structure and we have selected $l$ so that it is good (the hyperplanes are close together) for $h=2^{l}$ and (say) all $s \leq 16$ and $t \leq 32$. This was done for $51 \leq l \leq 150$ and we found that the structure was particularly good for $l=51,76$, and 127 .

\subsection{Precision and Speed of the RNG}

Note that the generator gives no more than 32 bits of precision even though it returns 53-bit floating-point numbers. If higher precision is required, successive numbers produced by the 


\section{Figure 1}

Overall arrangement of streams and substreams for the package.

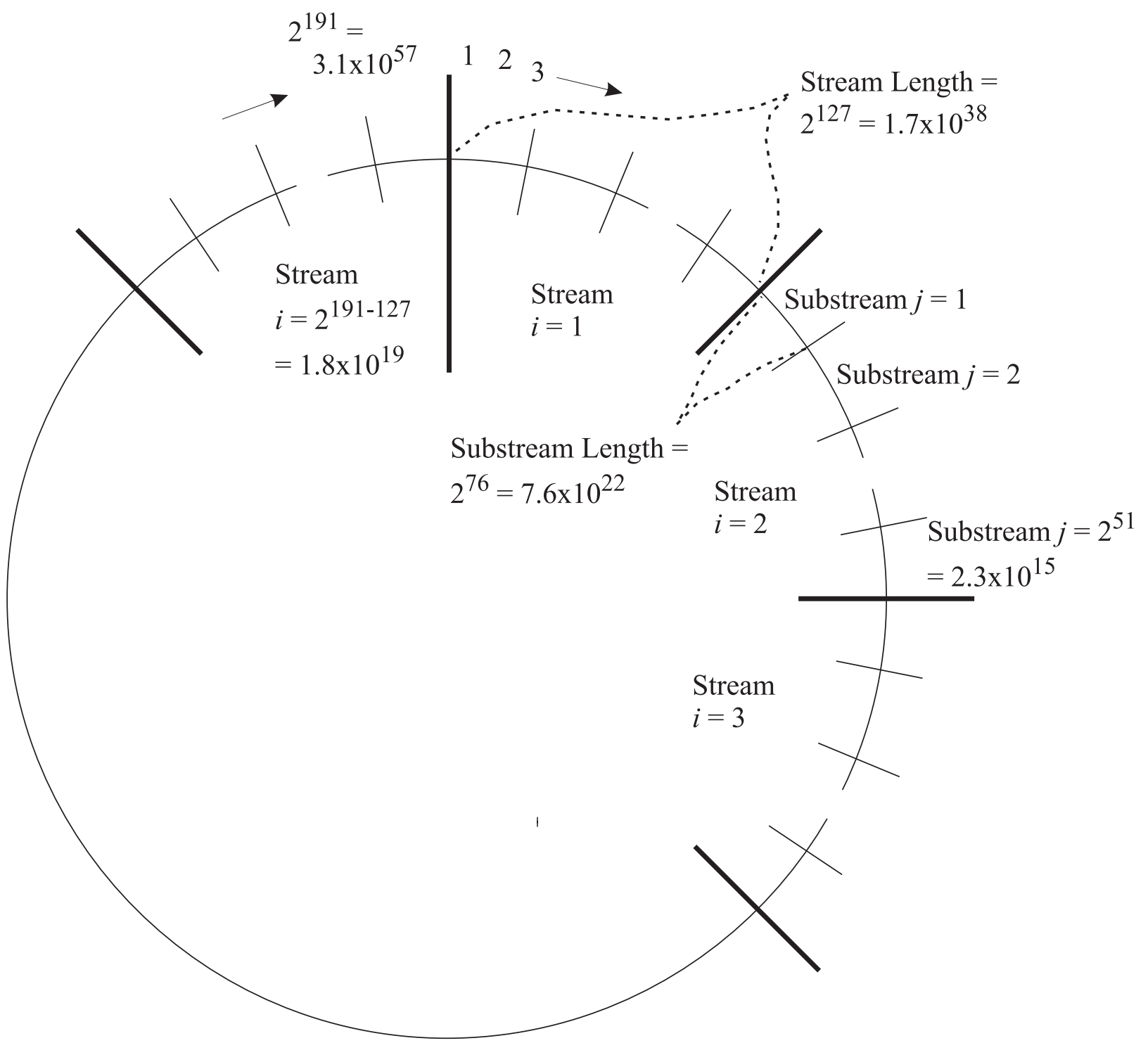




\section{Table II}

Timing reports.

\begin{tabular}{lrr}
\hline Function & $\begin{array}{r}\text { Time to generate } 10^{9} \\
\text { random numbers (minutes) }\end{array}$ & $\begin{array}{r}\text { Random numbers } \\
\text { generated per second }\end{array}$ \\
\hline & & \\
RandU01 & 12.9 & 1288659 \\
RandU01 (inc. prec.) & 28.5 & 585480 \\
RandInt & 18.5 & 901713 \\
lcgrand & 14.8 & 1127395 \\
\hline
\end{tabular}

generator may be used to construct each output value. That is, if the generator outputs the sequence $u_{1}, u_{2}, \ldots$, one can construct and use the sequence $v_{1}, v_{2}, \ldots$, defined by

$$
v_{i}=\left(v u_{2 i}+u_{2 i-1}\right) \bmod 1
$$

for some appropriate constant $v$, for instance $v=2^{-24}$.

Table II gives the total elapsed time (in minutes) for $10^{9}$ (one billion) calls to a given function from the package, and the number of random numbers generated per second for certain specific generators, on a Pentium III computer at $600 \mathrm{MHz}$ with $128 \mathrm{MB}$ of RAM, running Windows 98 and $\mathrm{C}++$ codes compiled with Microsoft visual $\mathrm{C}++$ version 6.0. To get an idea of the comparative speeds of the implementations, we also include the timing of LCG 1cgrand of Law and Kelton (2000, pp. 430-431). Note that lcgrand is implemented with integer arithmetic. Furthermore, these one billion random numbers represent almost half of the entire period of lcgrand, which would have been exhausted in about a half hour on our machine. The absolute times of generating the random numbers are extremely small and will be masked by other things going on in most simulations. If we use Moore's law assuming that computing speed doubles every 1.5 years, it will be approximately 219 years into the future before average desktop computers will have the capability to exhaust the cycle of RandU01 in a year of continuous computing.

\section{SUPPORT UTILITIES}

Some random-number packages offer a limited number of fixed streams, all based on the same generator, but using fixed starting seeds set, say, 100,000 values apart. This provides relatively low flexibility, as well as streams that are far too short (close together) for modern computers (even PCs). A simple procedure call should permit resetting a generator to 
a previous seed or jumping ahead to a new seed for the next run. Implementing such tools requires efficient jumping-ahead facilities, which in turn requires efficient procedures to compute the quantity $a \times s \bmod m$.

\subsection{Computing $a \times s \bmod m$}

If the product $a \times s \leq 2^{53}$, then it is always represented exactly in floating point on 32bit computers that support the IEEE-754 floating-point arithmetic standard, with at least 53 bits of precision for the mantissa. The generator can then be implemented directly in floating-point arithmetic, which is typically faster than an integer arithmetic implementation since it is done in hardware on the CPU. On the other hand, with this implementation, the state of the generator is represented over $64 k \times J$ bits, as opposed to $32 k \times J$ bits when the $x_{j, n}$ are represented as 32 -bit integers.

Now, consider a 32-bit computer on which all integers between $-2^{31}$ and $2^{31}$ (exclusive) are well represented. We want to compute $a \times s \bmod m$, where $a, s$, and $m$ are positive integers smaller than $2^{32}$. Without loss of generality, we assume that $a<m$ and $s<m$ (if not, replace $a$ and $s$ by $a \bmod m$ and $s \bmod m$, respectively). Performing the computation is tricky, because the product of $a \times s$ can exceed $2^{63}$, while double precision in most 32-bit computers carries no more than 53 bits of accuracy.

An algorithm was developed to compute $a \times s \bmod m$ with exact accuracy for the case where $a \times s>2^{53}$ by making sure that no operations in the algorithm produces a number greater than $2^{53}$. A direct approach, based on decomposition, operates as follows. Rewrite

$$
a=a_{1} \times 2^{17}+a_{2}
$$

SO

$$
a \times s=a_{1} \times s \times 2^{17}+a_{2} \times s .
$$

Therefore,

$$
a \times s \bmod m=\left(\left(a_{1} \times s \bmod m\right) \times 2^{17}+a_{2} \times s\right) \bmod m
$$

where $a_{1}<2^{15}$, so $a_{1} \times s<2^{47}\left(=2^{15} \times 2^{32}\right), a_{2}<2^{17}$, and $a_{2} \times s<2^{49}\left(=2^{17} \times 2^{32}\right)$. Because $v=\left(a_{1} \times s \bmod m\right)<2^{32}$, we have $v \times 2^{17}<2^{49}$ so that all the intermediate terms in the above computations are less than $2^{53}$. Therefore, all seed values will have exact accuracy. Refer to function MultModM in Appendix A for an implementation of this algorithm. Even 
though $a, s$, and $m$ are positive integers, they are declared (and represented) as double internally in our $\mathrm{C}++$ implementation.

\subsection{Computing $A^{v} \bmod m$}

The initial state of substreams can be computed easily if jumping-ahead facilities are available for the individual MRG components; that is, if an efficient algorithm is available for computing the state of the MRG $v$ steps ahead of the current one, for large values of $v$. L'Ecuyer (1990) explains one way of doing that, based on the fact that the MRG can be viewed as a LCG in matrix form, whose state is a $k$-dimensional vector and whose multiplier is a $k \times k$ matrix $A$. To jump ahead by $v$ values, just multiply the current state by $A^{v} \bmod m$. The matrix $A^{v} \bmod m$ can be pre-computed in time $\mathrm{O}(\log v)$, using the divide-and-conquer algorithm (Brassard and Bratley 1988, Knuth 1998, L'Ecuyer 1996).

That is, for the MRG, $s_{n+v}$ can be computed directly from $s_{n}$ using

$$
s_{n+v}=\left(A^{v} s_{n}\right) \bmod m=\left(A^{v} \bmod m\right) s_{n} \bmod m
$$

where

$$
A=\left(\begin{array}{cccc}
0 & 1 & \cdot & 0 \\
\cdot & \cdot & \cdot & \cdot \\
0 & 0 & \cdot & 1 \\
a_{k} & a_{k-1} & \cdot & a_{1}
\end{array}\right)
$$

When $A$ has this special structure, the first $k-1$ components of $s_{n}$ are obtained by shifting the last $k-1$ components of $s_{n-1}$, and the last component of $s_{n}$ is a linear combination of the components of $s_{n-1}$ according to the MRG recursion (L'Ecuyer 1990).

Thus, in our implementation the matrix $A_{1}$ for $s_{1, n}$ to jump ahead one step is

$$
A_{1}=\left(\begin{array}{ccc}
0 & 1 & 0 \\
0 & 0 & 1 \\
-810728 & 1403580 & 0
\end{array}\right)
$$

and the matrix $A_{2}$ for $s_{2, n}$ to jump ahead one step is

$$
A_{2}=\left(\begin{array}{ccc}
0 & 1 & 0 \\
0 & 0 & 1 \\
-1370589 & 0 & 527612
\end{array}\right) \text {. }
$$

The divide-and-conquer algorithm computes the jump-ahead matrix $A^{v}$ using the following recursion:

$$
A^{v} \bmod m= \begin{cases}A & \text { if } v=1 \\ A \times A^{v-1} \bmod m & \text { if } v>1, v \text { odd } \\ A^{v / 2} \times A^{v / 2} \bmod m & \text { if } v>1, v \text { even }\end{cases}
$$


See the procedure MatPowModM in Appendix A for an implementation of this algorithm for a $3 \times 3$ matrix.

\subsection{Jumping Backward}

Fermat's first theorem tells us that $A_{j}^{\rho_{j}} \bmod m_{j}=I$, where $\rho_{j}$ is the period length of the $j^{\text {th }}$ MRG component, because each component has a primitive characteristic polynomial. Therefore, $B_{j}=A_{j}^{\rho_{j}-1} \bmod m_{j}$ is the multiplicative inverse of $A_{j} \bmod m_{j}$. This means that $B_{j}$ is the jump-back-one-step matrix: Given a vector $s_{j, n}$, one can jump back $v$ steps to $s_{j, n-v}$ by multiplying $s_{j, n}$ by $B_{j}^{v}$. The matrix $B_{j}$ generates the same stream but in reverse order. As we can see from the new recursion below, the values of the parameters $b_{i j}$ are much larger than the original ones, where the $b_{i j}$ are the parameters of the new CMRG. Furthermore, we no longer have $b_{i j}\left(m_{j}-1\right)<2^{53}$ for all $b_{i j}$, so that the implementation would be slower than that of the original recursion.

The matrix $B_{1}$ for $s_{1, n}$ to jump back one step is

$$
B_{1}=\left(\begin{array}{ccc}
184888585 & 0 & 1945170933 \\
1 & 0 & 0 \\
0 & 1 & 0
\end{array}\right)
$$

and for $s_{2, n}$ to jump back one step we have

$$
B_{2}=\left(\begin{array}{ccc}
0 & 360363334 & 4225571728 \\
1 & 0 & 0 \\
0 & 1 & 0
\end{array}\right)
$$

The reverse stream follows the recursion

$$
\begin{aligned}
x_{1, n} & =\left(184888585 \times x_{1, n+1}+1945170933 \times x_{1, n+3}\right) \bmod 4294967087, \\
x_{2, n} & =\left(360366334 \times x_{2, n+2}+4225571728 \times x_{2, n+3}\right) \bmod 4294944443 \\
& =\left(360366334 \times x_{2, n+2}-69372715 \times x_{2, n+3}\right) \bmod 4294944443 .
\end{aligned}
$$

\section{A PORTABLE AND EFFICIENT PACKAGE FOR RANDOM-NUMBER GENERATION}

We now provide a set of portable utilities for random-number generation. The initial values of $\left(x_{1,0}, x_{1,1}, x_{1,2}\right)$ can be any non-negative integer values less than $m_{1}$ and not all zero, and the initial values of $\left(x_{2,0}, x_{2,1}, x_{2,2}\right)$ can be any non-negative integer values less than $m_{2}$ and not all zero. 
The initial seed of the main generator $s_{0}$ is the starting point of the first stream $I_{1}$. In the proposed package, the initial seed $s_{0}=I_{1}$ is set to the default value $(12345,12345,12345,12345$, 12345, 12345), but this value can be changed by the user (via SetPackageSeed). Each time a new RngStream object is created, its starting point (initial seed) $I_{g}$ is set $Z=2^{127}$ steps ahead of the starting point of the last created object. A vector named nextSeed is used to keep the seed values of the next created RngStream object (stream). For example, the declaration "RngStream g;" creates a stream with $I_{g}$ equal to nextSeed and advances nextSeed by $Z$ steps. Because the initial seed for each RngStream object is computed dynamically, no pre-computed list of seeds is needed.

For each RngStream, one can generate one value and go ahead one step, or go ahead to the beginning of the next substream within this stream, or go back to the beginning of the current substream, or to the beginning of the stream, or jump ahead or back by an arbitrary number of steps. As discussed in Section 1.1, the spacing between adjacent substreams is $W=2^{76}$. To get a feel for the extent of these spacings of streams and substreams, it would take over 1.8 billion years for RandU01 to exhaust one of the substreams using the hardware producing the timings of Table II. Once again assuming that Moore's law will continue to hold, RandU01 will require two months on an average desktop computer 50 years from now to exhaust one of the substreams (but over 385 millennia to exhaust one of the streams).

Several pre-computed jump matrices are provided in the RngStream class. With these jump matrices, we are able to compute the initial seed for each stream dynamically and reset the stream to various states. Thus, each instance of RngStream can assume different disjoint streams of which there are $2^{64} \approx 1.8 \times 10^{19}$ (so are virtually unlimited). Moreover, one can use AdvanceState to jump ahead or backward.

The methods Reset* reset a given stream either to its initial state $\left(C_{g} \leftarrow I_{g}\right.$ and $\left.B_{g} \leftarrow I_{g}\right)$, or to the beginning of its current substream $\left(C_{g} \leftarrow B_{g}\right)$, or to the beginning of its next substream $\left(C_{g} \leftarrow N_{g}\right.$ and $\left.B_{g} \leftarrow N_{g}\right)$. The method GetState returns the state of a stream. One can change the seed of a given stream, without modifying that of other streams, by invoking SetSeed or AdvanceState. However, after calling SetSeed for a given stream, the initial states of the different streams are no longer spaced $Z$ values apart. Therefore, this method should be used only in exceptional cases. The methods Reset* suffices for almost all applications.

The methods RandU01 and RandInt generate the uniform (pseudo)random numbers. 
Each stream can produce (if desired) antithetic random numbers with respect to the uniforms normally produced, i.e., return $1-U$ instead of $U$, by calling SetAntithetic, or produce 53-bit precision random numbers by calling IncreasePrecis.

Here are examples of situations where the tools offered in this package are useful:

- Compare two or more similar systems, via simulation with common random numbers, with $n$ simulation runs for each system. To guarantee that the same random numbers are used across the systems with good synchronization, assign different streams to different places where random numbers are needed in the model (e.g., to compare queuing systems, use one stream for interarrival times, one stream for the service times at each queue, one stream for a routing decision, etc.). To make sure that each stream starts from the same state across the different systems, assign run $j$ to the $j^{\text {th }}$ substream, within each stream. The experiment then proceeds as follows. For the first system, simulate run 1 by starting all the streams from their initial seed, $I_{g}$. Before each new run, advance all the streams to the initial state of their next substream, $N_{g}$. After the $n^{\text {th }}$ run, reset all the streams to their initial state, $I_{g}$. Repeat for each system to compare; see Example 2 in Section 4. Note that in this application, the substreams correspond to replications within each stream.

- Run simulations on several processors, in parallel, and each processor is to have its own (virtual) generator. In this case, simply use one stream for each processor. There must be a central authority, or monitor, to manage the allocation of the streams, but once a stream is allocated, the processors can go their own way, independently of each other. In this setup, the processors in fact use the same generator, but with different seeds. This makes the implementation much easier than if a different generator must be implemented on each processor.

We now describe the members of the $\mathrm{C}++$ class RngStream. This is the file RngStream.h, with comments.

\#include <string>

class RngStream

\{

public:

RngStream (const char *name = "");

This constructor creates a new stream with (optional) descriptor name. It initializes its seed $I_{g}$, and sets $B_{g}$ and $C_{g}$ to $I_{g}$. It also sets its anti and incPrec switches to false. The seed $I_{g}$ is 
equal to the initial seed of the package if this is the first stream created; otherwise it is $Z$ steps ahead of the seed of the most recently created stream.

static bool SetPackageSeed (const unsigned long seed[6]);

Sets the initial seed $s_{0}$ of the package to the six integers in the vector seed. The first 3 integers in the seed must all be less than $m_{1}=4294967087$, and not all 0 ; and the last 3 integers must all be less than $m_{2}=4294944443$, and not all 0 . If this method is not called, the default initial seed is $(12345,12345,12345,12345,12345,12345)$. Returns false for invalid seeds, and true otherwise.

void ResetStartStream ();

Reinitializes the stream to its initial state: $C_{g}$ and $B_{g}$ are set to $I_{g}$.

void ResetStartSubstream ();

Reinitializes the stream to the beginning of its current substream: $C_{g}$ is set to $B_{g}$.

void ResetNextSubstream ();

Reinitializes the stream to the beginning of its next substream: $N_{g}$ is computed, and $C_{g}$ and $B_{g}$ are set to $N_{g}$.

void SetAntithetic (bool a);

If $\mathrm{a}=$ true, the stream will start generating antithetic variates, i.e., $1-U$ instead of $U$, until this method is called again with a $=$ false.

void IncreasedPrecis (bool incp);

After calling this method with incp = true, each call to the generator (direct or indirect) for this stream will return a uniform random number with more bits of resolution (53 bits if machine follows IEEE 754 standard) instead of 32 bits, and will advance the state of the stream by 2 steps instead of 1 . More precisely, if $\mathbf{s}$ is a stream of the class RngStream, in the nonantithetic case, the instruction " $\mathrm{u}=\mathrm{s}$.RandU01()" will be equivalent to " $\mathrm{u}=$ ( $\mathrm{s}$. RandU01()

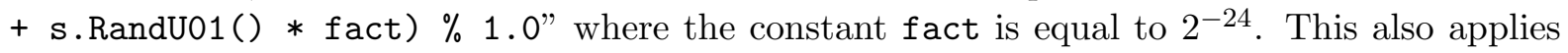
when calling RandU01 indirectly (e.g., via RandInt, etc.). By default, or if this method is called again with incp $=$ false, each call to RandU01 for this stream advances the state by 1 step and returns a number with 32 bits of resolution.

bool SetSeed (const unsigned long seed[6]);

Sets the initial seed $I_{g}$ of the stream to the vector seed. The vector seed should contain valid seed values as described in SetPackageSeed. The state of the stream is then reset to this initial seed. The states and seeds of the other streams are not modified. As a result, after calling this method, the initial seeds of the streams are no longer spaced $Z$ values apart. We discourage the use of this method; proper use of the Reset* methods is preferable. Returns false for invalid seeds, and true otherwise.

void AdvanceState (long e, long c);

Advances the state by $n$ steps (see below for the meaning of $n$ ), without modifying the states of other streams or the values of $B_{g}$ and $I_{g}$ in the current object. If e $>0$, then $n=2^{\mathrm{e}}+\mathrm{c}$; if $\mathrm{e}<0$, then $n=-2^{-\mathrm{e}}+\mathrm{c}$; and if $\mathrm{e}=0$, then $n=\mathrm{c}$. Note: c is allowed to take negative values. We discourage the use of this method.

void GetState (unsigned long seed[6]) const;

Returns in seed [0..5] the current state $C_{g}$ of this stream. This is convenient if we want to 
save the state for subsequent use.

void WriteState () const;

Writes (to standard output) the current state $C_{g}$ of this stream.

void WriteStateFull () const;

Writes (to standard output) the value of all the internal variables of this stream: name, anti, incPrec, $\mathrm{Ig}, \mathrm{Bg}, \mathrm{Cg}$.

double RandU01 ();

Normally, returns a (pseudo)random number from the uniform distribution over the interval $(0,1)$, after advancing the state by one step. The returned number has 32 bits of precision in the sense that it is always a multiple of $1 /\left(2^{32}-208\right)$. However, if IncreasedPrecis (true) has been called for this stream, the state is advanced by two steps and the returned number has 53 bits of precision.

int RandInt (int $i$, int $j$ );

Returns a (pseudo)random number from the discrete uniform distribution over the integers $\{i, i+1, \ldots, j\}$. Makes one call to RandU01.

private:

double $\mathrm{Cg}[6], \operatorname{Bg}[6], \operatorname{Ig}[6]$;

Vectors to store the current seed, the beginning of the current block (substream) and the beginning of the current stream.

bool anti, incPrec;

Variables to indicate whether to generate antithetic or increased precision random numbers.

std: :string name;

String to store the optional name of the current RngStream object.

static double nextSeed [6];

Static vector to store the beginning state of the next RngStream to be created (instantiated).

double U01 ();

The backbone uniform random number generator.

double U01d ();

The backbone uniform random number generator with increased precision.

\};

\section{EXAMPLES}

Example1.c in Figure 2 shows how we generate a list of ten seed vectors. We set the package seed to $\{327612383,317095578,14704821,884064067,1017894425,16401881\}$ by calling SetPackageSeed before instantiating any RngStream object. Note that if SetPackageSeed is not executed, $\{12345,12345,12345,12345,12345,12345\}$ will be used as the default 
package seed. The declaration "RngStream RngObj" is inside the for loop on $i$. Therefore, the instance of the object dies before the next iteration, i.e., the object RngObj is not available outside the for loop on $i$. Each declaration will create the RngObj instance with $I_{g}=B_{g}=C_{g}=$ nextSeed and advance nextSeed by $2^{127}$ steps. Thus, the output seeds will be $2^{127}$ apart.

Figure 2

example1.C.

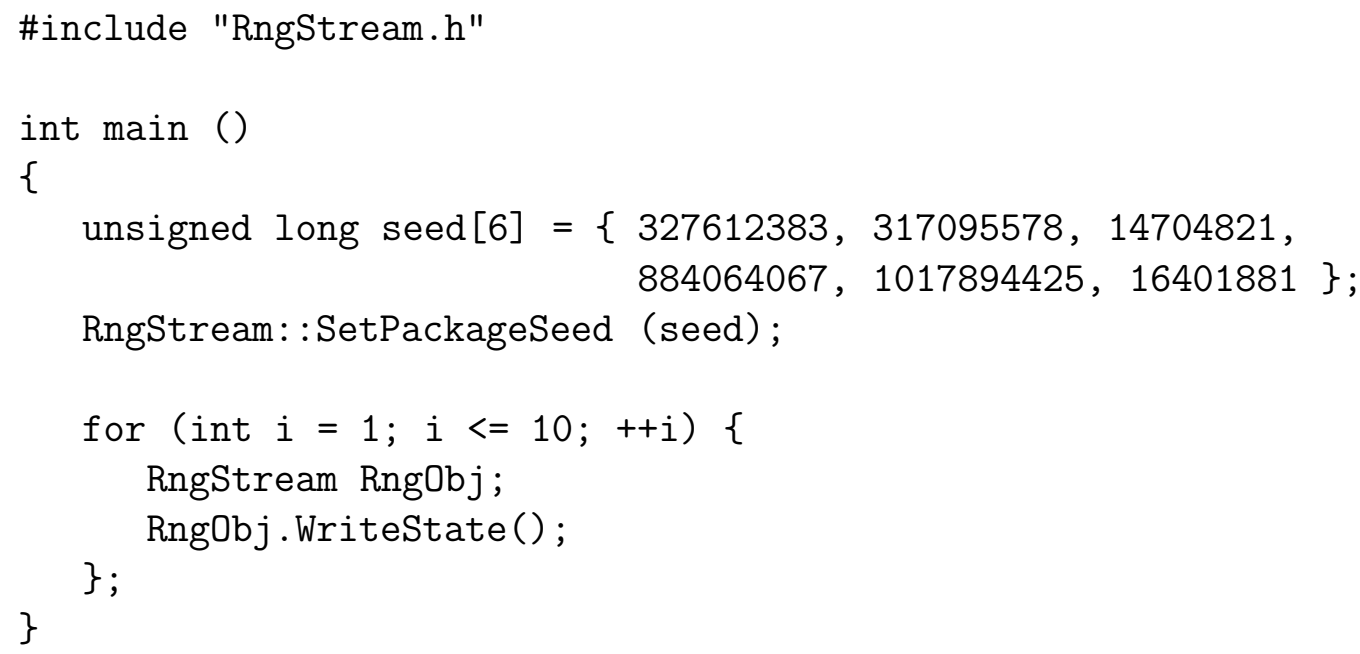

Example2.c in Figure 3 shows how to apply some of the utilities supplied in the package. The declarations "RngStream RngObj1" and "RngStream RngObj2" will create the RNG objects with

$$
I_{g}=B_{g}=C_{g}=\{12345,12345,12345,12345,12345,12345\}
$$

and

$$
I_{g}=B_{g}=C_{g}=\{3692455944,1366884236,2968912127,335948734,4161675175,475798818\}
$$

respectively, i.e., $2^{127}$ steps apart. The first RNG object RngObj1 may be dedicated to generate interarrival times while the second RNG object RngObj2 may be dedicated to generate service times, for some queueing system to be simulated. We generate five interarrival times and five service times, then move each RNG to its next substream. This is repeated ten times, thus yielding ten vectors, each containing five interarrival times and five service times. Moreover, these ten vectors will be exactly the same at iterations $k=0$ and $k=1$ of the outer for loop, because the statements "RngObj1. ResetStartStream" and "RngObj2. ResetStartStream" will reset the current seeds $C_{g}$ of both RNG objects to the initial seed $I_{g}$. 


\section{Figure 3}

\#include <math.h>

example2.C.

\#include "RngStream.h"

int main ()

\{

RngStream RngObj1;

RngStream RngObj2;

double interArrival;

double serviceTime;

for (int $\mathrm{k}=0 ; \mathrm{k}<=1 ;++\mathrm{k})\{$

for (int $j=1 ; j<=10 ;++j$ ) \{

for (int $i=1$; $i<=5$; ++i) \{

interArrival $=-\log (1.0-$ RngObj1.RandU01 ()$)$;

serviceTime $=-0.9 * \log (1.0-\operatorname{RngObj} 2 \cdot \operatorname{RandU01}())$;

\};

RngObj1.ResetNextSubstream ();

RngObj2.ResetNextSubstream ();

\};

RngObj1.ResetStartStream ();

RngObj2.ResetStartStream ();

\};

\}

\section{SUMMARY}

We have discussed the backbone CMRG random-number generator, several utilities to enhance its practical use, and implementation issues. We briefly mentioned how the spacing between each stream was chosen, based on a lattice-structure analysis of its successive output values. We implemented a $\mathrm{RNG}$ package in the $\mathrm{C}++$ language. Our implementation eliminates the need to use a fixed number of pre-computed seeds, which would provide little flexibility. The proposed RNG package provides jumping facilities, has good speed, a long period, and excellent theoretical/statistical properties.

\section{ACKNOWLEDGMENTS}

This work has been supported by NSERC-Canada grant number ODGP0110050 to the first author. We thank C. Dennis Pegden for suggesting the type of depiction in Figure 1. 


\section{REFERENCES}

Brassard, G and P. Bratley. 1988. Algorithmics, Theory and Practice. Prentice-Hall.

Knuth, D. E. 1998. The Art of Computer Programming, Volume 2: Seminumerical Algorithms. Third ed. Reading, Mass.: Addison-Wesley.

Law, A. M and W. D. Kelton. 2000. Simulation Modeling and Analysis. Third ed. New York: McGraw-Hill.

L'Ecuyer, P. 1990. Random numbers for simulation. Communications of the ACM, 33(10), 85-97.

L'Ecuyer, P. 1996. Combined multiple recursive random number generators. Operations Research, 44(5), 816-822.

L'Ecuyer, P. 1999a. Good parameters and implementations for combined multiple recursive random number generators. Operations Research, 47(1), 159-164.

L'Ecuyer, P. 1999b. Tables of maximally equidistributed combined LFSR generators. Mathematics of Computation, 68(225), 261-269.

L'Ecuyer, P and T. H. Andres. 1997. A random number generator based on the combination of four LCGs. Mathematics and Computers in Simulation, 44, 99-107.

L'Ecuyer, P. and S. Côté. 1991. Implementing a random number package with splitting facilities. ACM Transactions on Mathematical Software, 17(1), 98-111.

L'Ecuyer, P. and R. Simard. 2001. On the performance of birthday spacings tests for certain families of random number generators. Mathematics and Computers in Simulation, 55(1-3), 131-137.

L'Ecuyer, P., R. Simard, E. J. Chen, and W. D. Kelton. 2001. An object-oriented randomnumber package with many long streams and substreams. Submitted.

Marsaglia, G. 1985. A current view of random number generators. In Computer Science and Statistics, Sixteenth Symposium on the Interface, 3-10, North-Holland, Amsterdam. Elsevier Science Publishers.

Mascagni, M and A. Srinivasan. 2000. Algorithm 806: SPRNG: A scalable library for pseudorandom number generation. ACM Transactions on Mathematical Software, 26, 436-461.

Matsumoto, M. and T. Nishimura. 1998. Mersenne twister: A 623-dimensionally equidis- 
tributed uniform pseudo-random number generator. ACM Transactions on Modeling and Computer Simulation, 8(1), 3-30. 
APPENDIX A. A C ++ IMPLEMENTATION

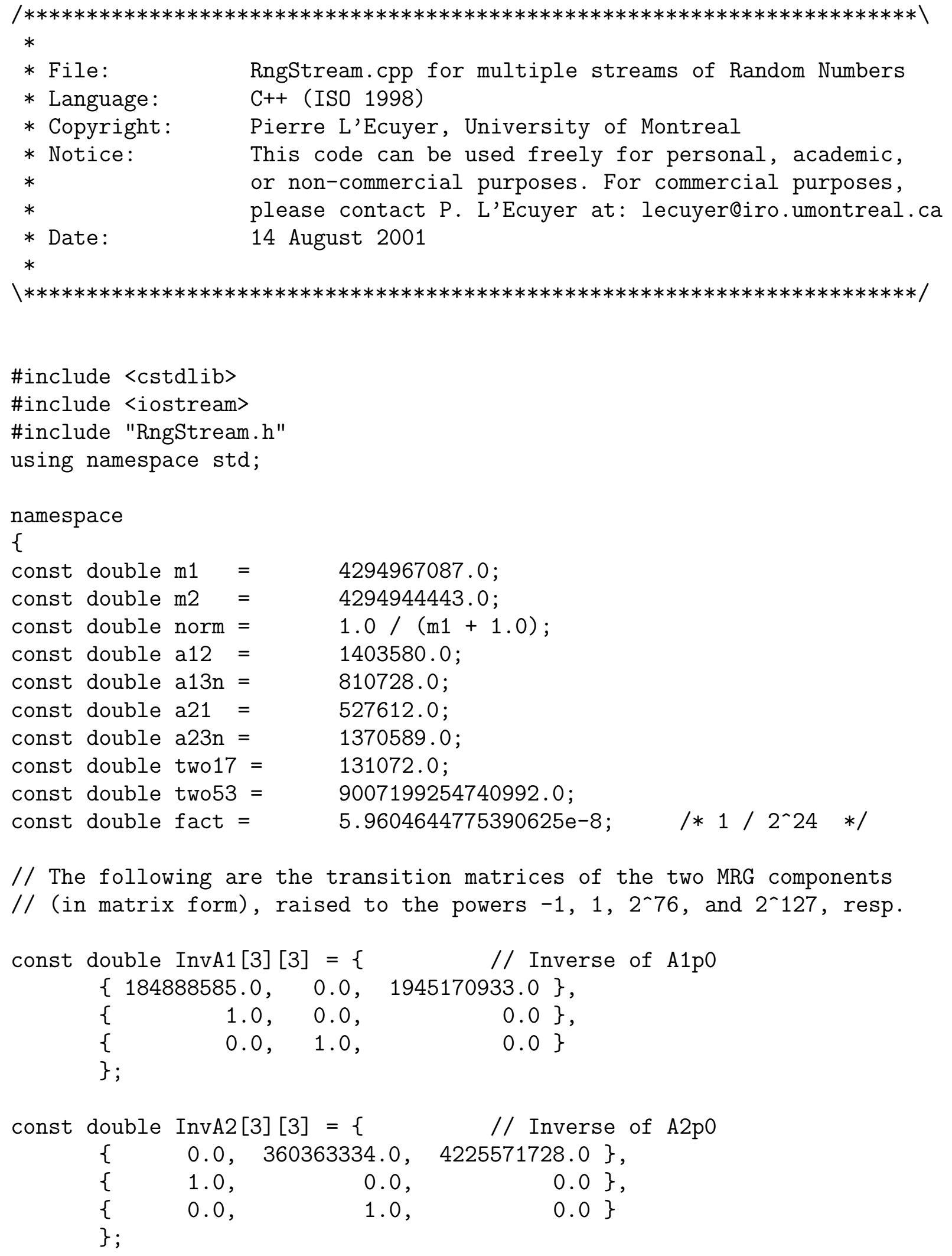




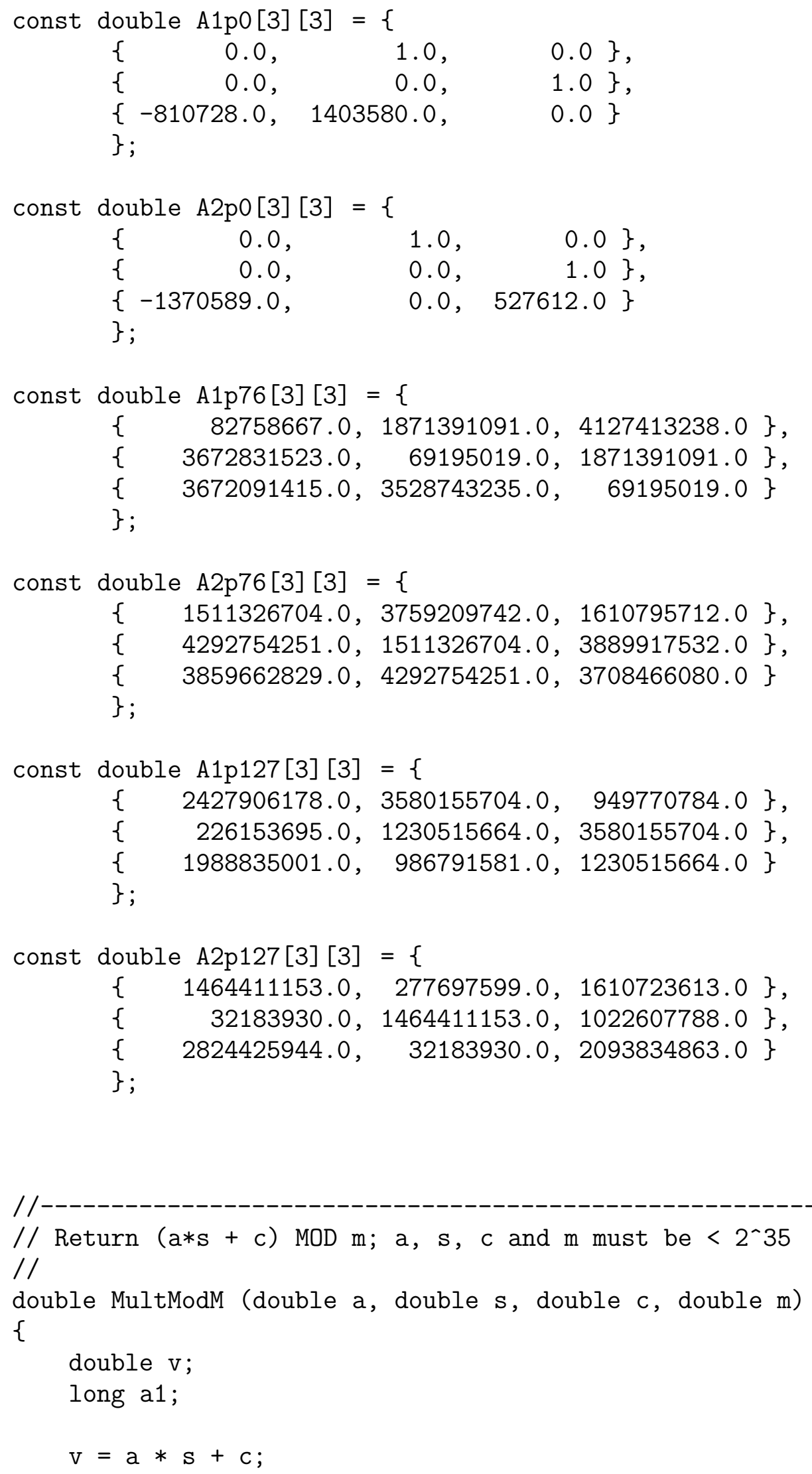




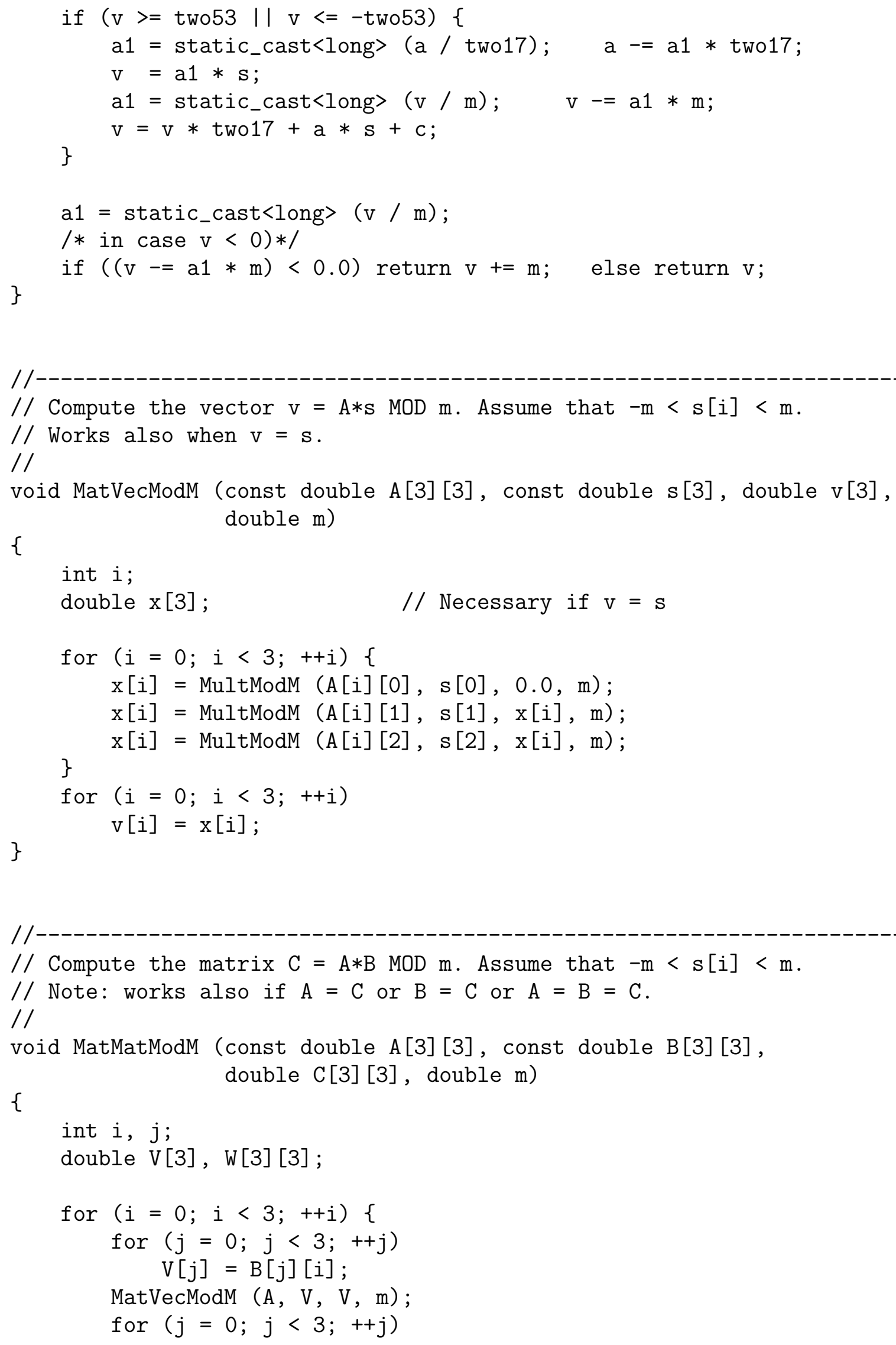




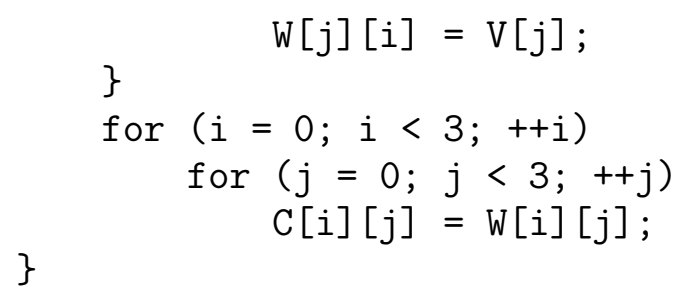




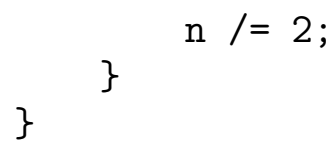

// Check that the seeds are legitimate values. Returns 0 if legal seeds, // -1 otherwise.

//

int CheckSeed (const unsigned long seed[6])

\{

int $i$;

for $(i=0 ; i<3 ;++i)\{$

if (seed $[\mathrm{i}]>=\mathrm{m} 1)\{$

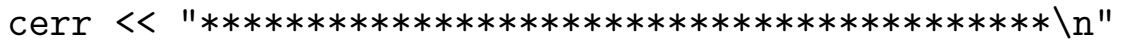
<< "ERROR: Seed[" $<$ i $<$ "] > 4294967087 , Seed is not set." $<<" \backslash \mathrm{n} * * * * * * * * * * * * * * * * * * * * * * * * * * * * * * * * * * * * * * * * \backslash \mathrm{n} \backslash \mathrm{n} "$; return $(-1)$;

\}

\}

for $(i=3$; $i<6$; ++i) \{

if ( seed $[i]>=\mathrm{m} 2)\{$

cerr < " $* * * * * * * * * * * * * * * * * * * * * * * * * * * * * * * * * * * * * * * * * \backslash \mathrm{n} "$

<< "ERROR: Seed[" $<$ i $<$ "] > 4294944443 , Seed is not set."

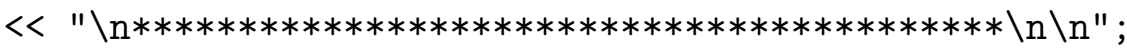
return $(-1)$;

\}

\}

if $(\operatorname{seed}[0]==0$ \&\& $\operatorname{seed}[1]==0$ \&\& $\operatorname{seed}[2]==0)\{$

cerr $<<* * * * * * * * * * * * * * * * * * * * * * * * * * * * \backslash \mathrm{n} "$

$<$ "ERROR: First 3 seeds $=0 . \backslash \mathrm{n}$ "

$<<" * * * * * * * * * * * * * * * * * * * * * * * * * * * * \backslash \mathrm{n} \backslash \mathrm{n} "$;

return $(-1)$;

\}

if $(\operatorname{seed}[3]==0$ \&\& $\operatorname{seed}[4]==0 \& \& \operatorname{seed}[5]==0)\{$

cerr << "******************************\n"

$<$ "ERROR: Last 3 seeds $=0 . \backslash \mathrm{n}$ "

$<<" * * * * * * * * * * * * * * * * * * * * * * * * * * * * \backslash \mathrm{n} \backslash \mathrm{n} "$;

return $(-1)$;

\}

return 0 ;

\}

\} // end of anonymous namespace 


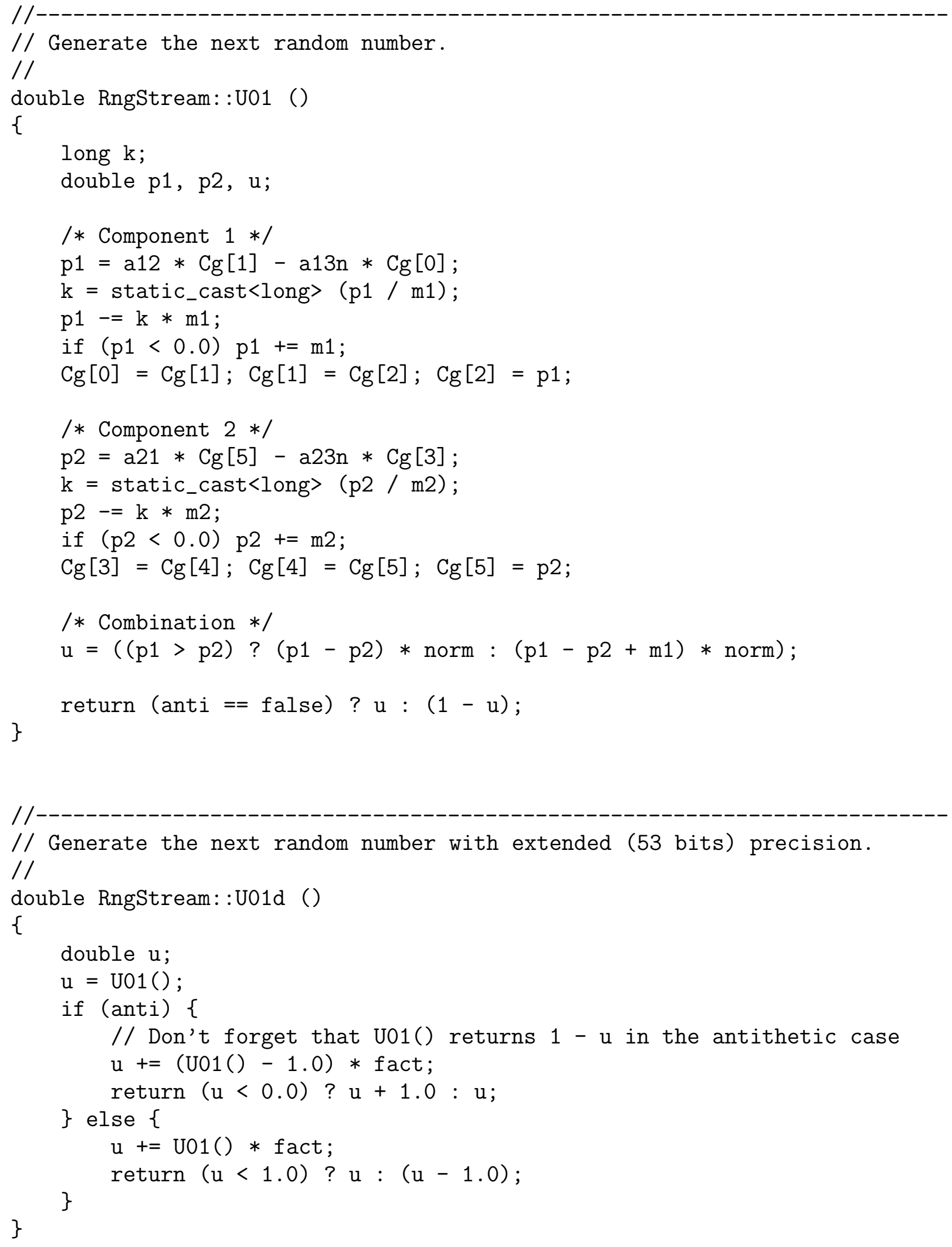

$/ / * * * * * * * * * * * * * * * * * * * * * * * * * * * * * * * * * * * * * * * * * * * * * * * * * * * * * * * * * * * * * * * * * * * * * * * * *$ 
// Public members of the class start here

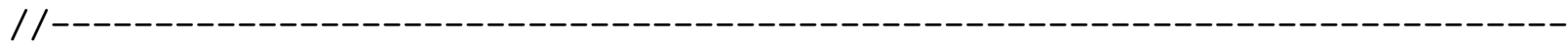

// The default seed of the package; will be the seed of the first

// declared RngStream, unless SetPackageSeed is called.

//

double RngStream: : nextSeed [6] =

\{

$12345.0,12345.0,12345.0,12345.0,12345.0,12345.0$

\};

$1 /$

// constructor

//

RngStream: :RngStream (const char *s) : name (s)

\{

anti $=$ false;

incPrec $=$ false;

/* Information on a stream. The arrays $\{\mathrm{Cg}, \mathrm{Bg}, \mathrm{Ig}\}$ contain the current state of the stream, the starting state of the current SubStream, and the starting state of the stream. This stream generates antithetic variates if anti = true. It also generates numbers with extended precision (53 bits if machine follows IEEE 754 standard) if incPrec $=$ true. nextSeed will be the seed of the next declared RngStream. */

for (int $i=0 ; i<6$; ++i) \{

\}

$\mathrm{Bg}[\mathrm{i}]=\mathrm{Cg}[\mathrm{i}]=\operatorname{Ig}[\mathrm{i}]=\operatorname{nextSeed}[\mathrm{i}]$;

MatVecModM (A1p127, nextSeed, nextSeed, m1);

MatVecModM (A2p127, \&nextSeed [3], \&nextSeed [3], m2); \}

// Reset Stream to beginning of Stream.

//

void RngStream: :ResetStartStream ()

\{

for (int $i=0 ; i<6 ;++i)$

$\operatorname{Cg}[i]=\operatorname{Bg}[i]=\operatorname{Ig}[i]$;

\}

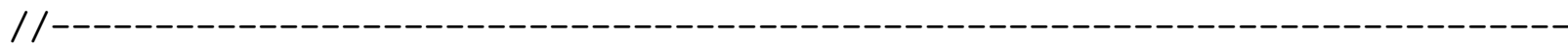


// Reset Stream to beginning of SubStream.

/I

void RngStream: :ResetStartSubstream ()

\{

for (int $i=0 ; i<6 ;++i$ )

\}

$\mathrm{Cg}[\mathrm{i}]=\mathrm{Bg}[\mathrm{i}]$

// Reset Stream to NextSubStream.

//

void RngStream: :ResetNextSubstream ()

\{

MatVecModM (A1p76, $\mathrm{Bg}, \mathrm{Bg}, \mathrm{m} 1)$;

MatVecModM(A2p76, \&Bg[3], \&Bg[3], m2);

for (int $i=0 ; i<6 ;++i$ )

\}

$\mathrm{Cg}[\mathrm{i}]=\mathrm{Bg}[\mathrm{i}]$;

bool RngStream: : SetPackageSeed (const unsigned long seed[6])

\{

if (CheckSeed (seed)) return false;

// FAILURE

for (int $i=0 ; i<6 ;++i$ ) nextSeed $[i]=\operatorname{seed}[i]$;

return true;

// SUCCESS

\}

bool RngStream: :SetSeed (const unsigned long seed[6])

\{

if (CheckSeed (seed)) return false;

// FAILURE

for (int $i=0 ; i<6 ;++i)$

$\mathrm{Cg}[\mathrm{i}]=\mathrm{Bg}[\mathrm{i}]=\operatorname{Ig}[\mathrm{i}]=\operatorname{seed}[\mathrm{i}]$;

return true;

// SUCCESS

\}

$/ /-$

$/ /$ if $e>0$, let $\mathrm{n}=2^{\wedge} \mathrm{e}+\mathrm{c}$;

$/ /$ if $e<0$, let $n=-2^{\wedge}(-e)+c$;

$/ /$ if $e=0$, let $n=c$.

// Jump $\mathrm{n}$ steps forward if $\mathrm{n}>0$, backwards if $\mathrm{n}<0$. 


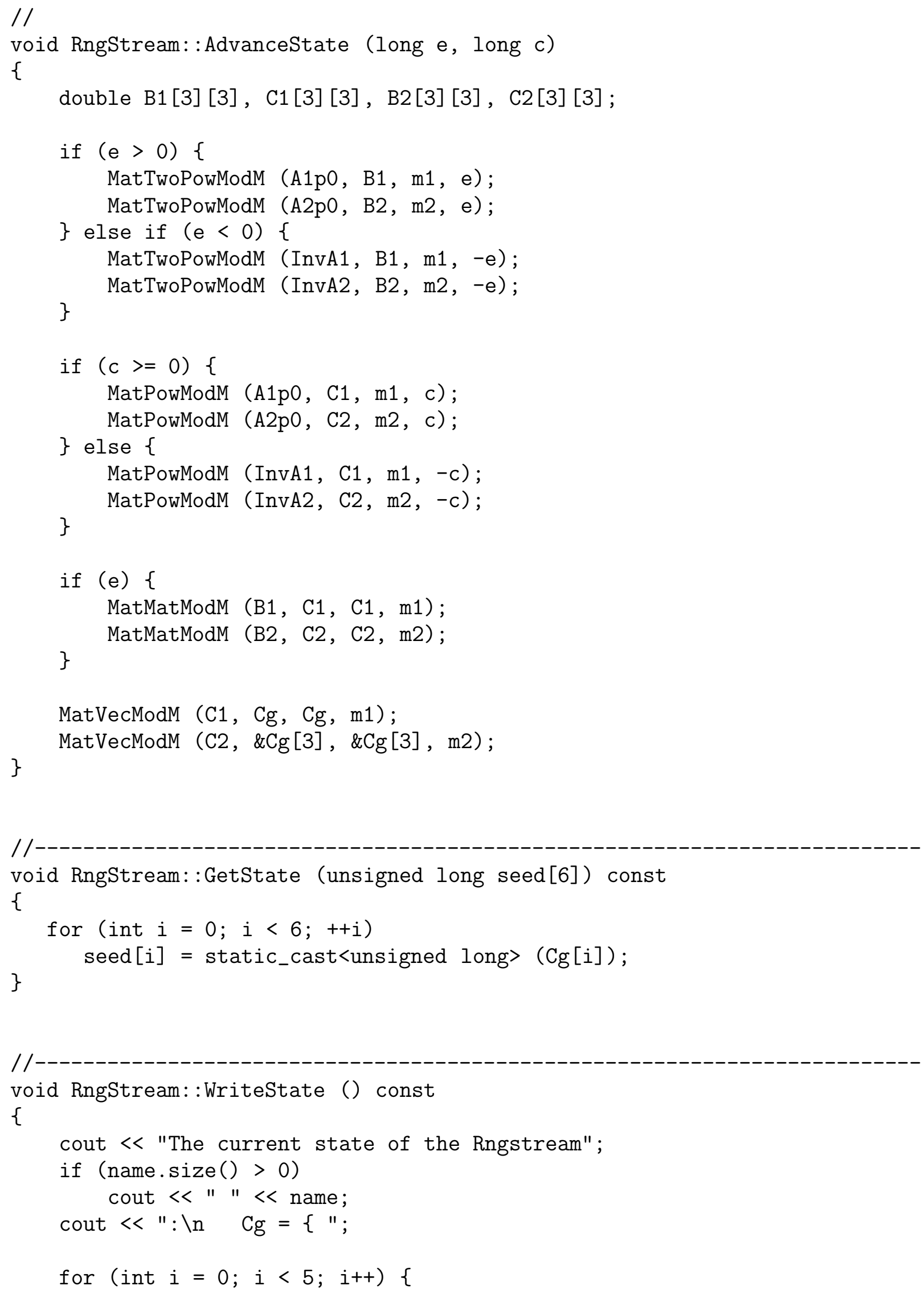




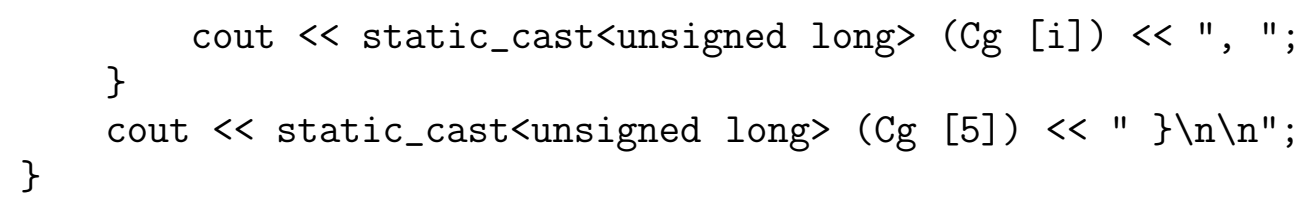


//-------------------------------------------------------------------------

// Generate the next random number.

/I

double RngStream: :RandU01 ()

\{

if (incPrec) return U01d();

else return $\mathrm{U01}()$;

\}

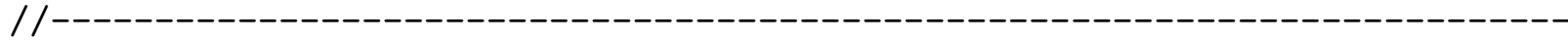

// Generate the next random integer.

/I

int RngStream: RandInt (int low, int high)

\{

return low + static_cast<int> ( high - low + 1.0) * RandU01 ());

\} 